01.5

\title{
Спектры нормальных мод иерархических ансамблей взаимосвязанных осцилляторов
}

\author{
() О.С. Сафиина ${ }^{1,2}$, А.В. Воронов ${ }^{1}$, А.Р. Сафинн ${ }^{1,3}$, М.Ф. Булатов ${ }^{2}$, Д.В. Чуриков ${ }^{2,3}$, Е.Д. Суровяткина ${ }^{4,5}$ \\ ${ }^{1}$ Национальный исследовательский университет „МЭИ“, Москва, Россия \\ ${ }^{2}$ Научно-технологический центр уникального приборостроения РАН, Москва, Россия \\ ${ }^{3}$ Институт радиотехники и электроники им. В.А. Котельникова РАН, Москва, Россия \\ ${ }^{4}$ Институт космических исследований РАН, Москва, Россия \\ ${ }^{5}$ Потсдамский институт исследования климатических изменений, Потсдам, Германия \\ E-mail: arnellemorte@gmail.com
}

Поступило в Редакцию 24 декабря 2018 г.

В окончательной редакции 24 декабря 2018 г.

Принято к публикации 29 мая 2019 г.

\begin{abstract}
Построено семейство спектров нормальных мод иерархически организованных ансамблей идентичных взаимосвязанных осцилляторов с различной топологией организации. Показано, что древовидные ансамбли обладают фрактальным спектром нормальных мод типа „чертова лестница“, причем с ростом количества ветвей дерева и при введении дополнительных связей между элементами ансамбля количество вырожденных мод возрастает. Приведен анализ влияния топологий ансамблей и сил связей между его элементами на спектральные характеристики.
\end{abstract}

Ключевые слова: осцилляторы, нормальные моды, нелинейные колебания, фракталы.

DOI: 10.21883/PJTF.2019.17.48219.17651

Теория сетей взаимосвязанных нелинейных осцилляторов в настоящее время интенсивно развивается [1] в связи с возросшими вычислительными возможностями современных компьютеров и совершенствованием методов моделирования. Несмотря на большое количество работ по динамическим сетям, некоторые важные характеристики таких сетей изучены слабо. В частности, ввиду сложности моделирования практически неисследованной остается задача о влиянии топологии сети на ее динамические свойства. Чаще всего в простейших сетях исследуются различные динамические процессы: синхронизация, многомодовые колебания, солитоны и бризеры, химерные состояния [2]. Подробно исследованы вопросы синхронизации и устойчивости нормальных мод взаимосвязанных осцилляторов ван дер Поля, расположенных в ансамблях в виде линейки [3], двумерной решетки [4], кольца [5] и цилиндра [6], однако в этом аспекте практически не рассматриваются более сложно организованные сети. Вместе с тем природные сложно организованные сети, такие как головной мозг млекопитающих, совокупность генов в ДНК и многие другие, обладают чрезвычайно сложной топологией, которой определяется значительное количество характеристик сетей.

Во многих сложных сетях можно выделить определенную иерархию и самоподобие [7]. Соответствующие сети называются ультраметрическими [8], т.е. обладающими усиленной метрикой (сильное неравенство треугольника). Применение ультраметрического анализа для определенного класса сложных систем позволяет перейти от описания энергетических ландшафтов, содержащих большое число локальных энергетических минимумов, к иерархическим бассейнам локальных минимумов. Другими словами, стандартная задача о нахождении собст- венных значений матриц таких систем сводится к решению одного уравнения ультраметрической диффузии. Этот подход активно применяется для анализа динамики сложных белковых соединений, молекулярных машин, спиновых стекол и т.д. $[9,10]$. В частности, локальные высокочастотные движения отдельных частей молекулярной машины могут приводить к низкочастотным движениям всей структуры. При этом энергия высокочастотных колебаний частично переходит в энергию низкочастотных колебаний, что может быть использовано для управления свойствами молекулярной машины. В связи с этим возникает интерес к исследованию свойств иерархических сетей, в том числе в случае, когда отдельный элемент сети представляется не одним осциллятором, а определенной структурой осцилляторов (назовем их метаструктурами). Целью настоящей работы является исследование нормальных мод иерархически организованных ансамблей идентичных взаимосвязанных осцилляторов с различной топологией организации, а также влияния топологии метаструктур на вид их спектра.

Рассмотрим $N$ взаимосвязанных осцилляторов, которые можно характеризовать вектором $\mathbf{x}(t)=\left[x_{1}(t), \ldots, x_{N}(t)\right]^{T}$, где $x_{i}(t)-$ искомые функции времени парциального осциллятора, [o] $]^{T}$ символ транспонирования. Будем считать, что осцилляторы консервативны и связаны линейными связями так, что их динамику можно описать вторым законом Ньютона

$$
d^{2} \mathbf{x} / d t^{2}=\mathbf{F}\left[x_{1}, \ldots, x_{N}\right],
$$

где компоненты вектора $\mathbf{F}$ равны $F_{i}=-\partial U / \partial x_{i}$, а функция $U=\sum_{i=1}^{N} \sum_{j=1}^{N} \alpha_{i j} x_{i} x_{j}-$ потенциальная энергия 

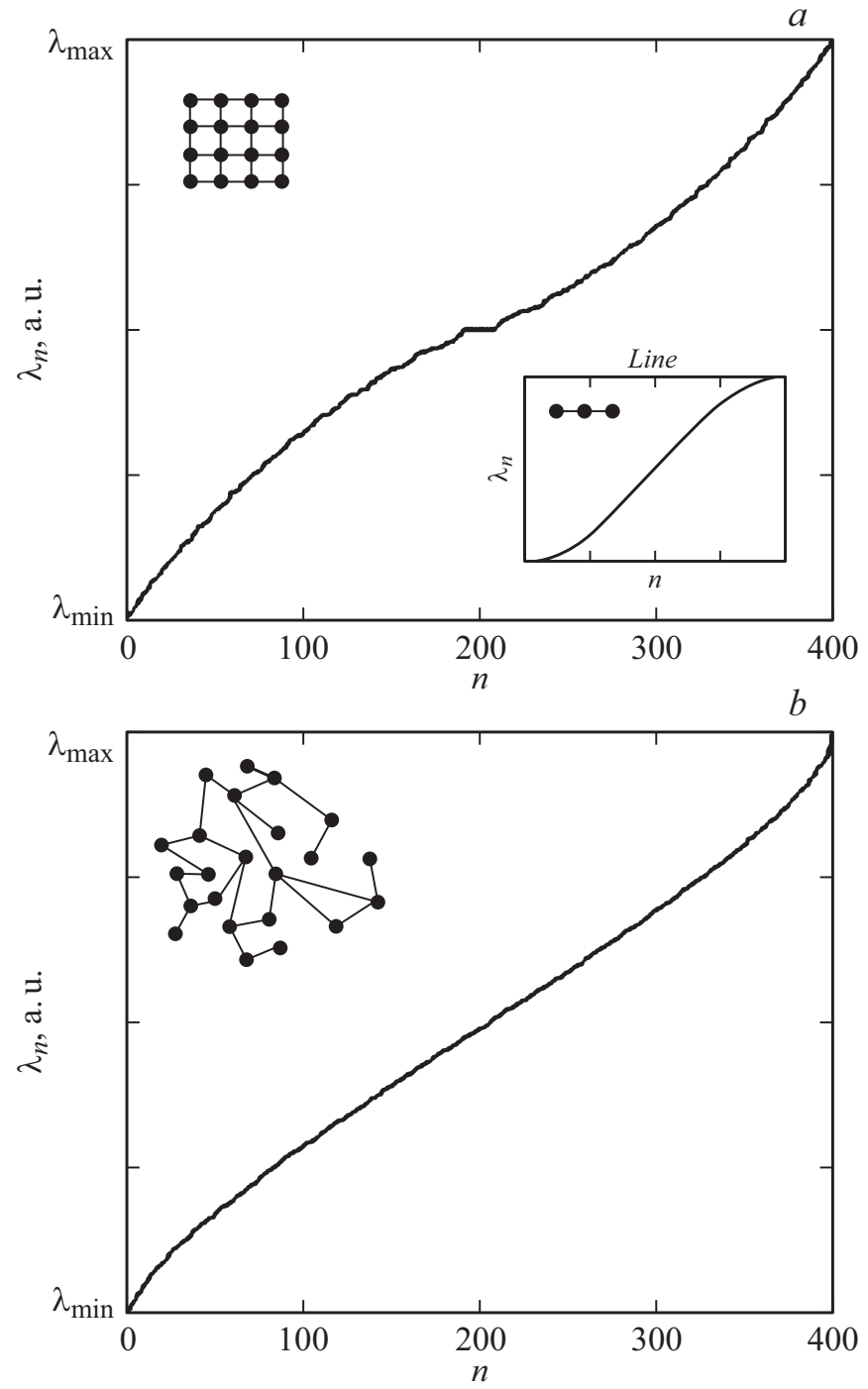

Рис. 1. Спектры нормальных мод $\lambda_{n}$ двумерной решетки $(a)$ и случайно организованной сети $(b)$ взаимосвязанных осцилляторов. На вставке изображен спектр линейки.

системы, представимая в виде стандартной квадратичной формы, $\alpha_{i j}$ - элементы матрицы связей $\Omega i$-го элемента с $j$-м. Уравнение (1) можно представить в следующем виде:

$$
d^{2} \mathbf{x} / d t^{2}+\Omega \cdot \mathbf{x}=0
$$

что является многомерной записью уравнения линейного осциллятора. Уравнение (2) для любой сколь угодно большой матрицы $\Omega$ имеет набор собственных чисел $\lambda_{i}$, представляющих собой нормальные частоты колебаний. Уравнение для определения нормальных частот имеет стандартный вид (см., например, [6])

$$
\operatorname{det}\left[\Omega-\operatorname{diag}\left(\lambda_{1}^{2}, \ldots, \lambda_{N}^{2}\right)\right]=0 .
$$

Как известно [3-5], количество нормальных мод колебаний равно количеству осцилляторов, но часть из них может быть вырожденной (т.е. соответствующие нормальные частоты совпадают). Сделаем переход к новым координатам, для которых уравнения консервативной системы уже не будут связанными. Для этого введем вектор $\mathbf{y}=P \cdot \mathbf{x}$, где $P$ - матрица коэффициентов распределения. Уравнения консервативной системы в новых координатах $\mathbf{y}=\left[y_{1}, \ldots, y_{N}\right]^{T}$ будут иметь вид

$$
\frac{d^{2} y_{n}}{d t^{2}}+\lambda_{n}^{2} y_{n}=0
$$

Далее рассмотрим спектр нормальных частот для различных топологий сетей (различных матриц $\Omega$ ). В первую очередь представим спектры наиболее широко изученных ансамблей осцилляторов: линейки и двумерной решетки. Также в этот список добавим сеть, распределенную случайным образом. Спектры нормальных мод перечисленных сетей приведены на рис. 1. Видно, что спектры линейки и случайно распределенной сети не имеют вырожденных мод, тогда как в спектре двумерной решетки имеется узкая область, соответствующая определенному значению вырожденной моды. При этом вид спектра случайно организованной сети зависит от закона распределения параметров отдельных осцилляторов. Сети, обладающие богатым спектром нормальных мод, хорошо подходят для применения в многочастотных коммуникационных системах благодаря возможности широкой перестройки. Синхронизация в таких системах возможна только на соседних частотах.
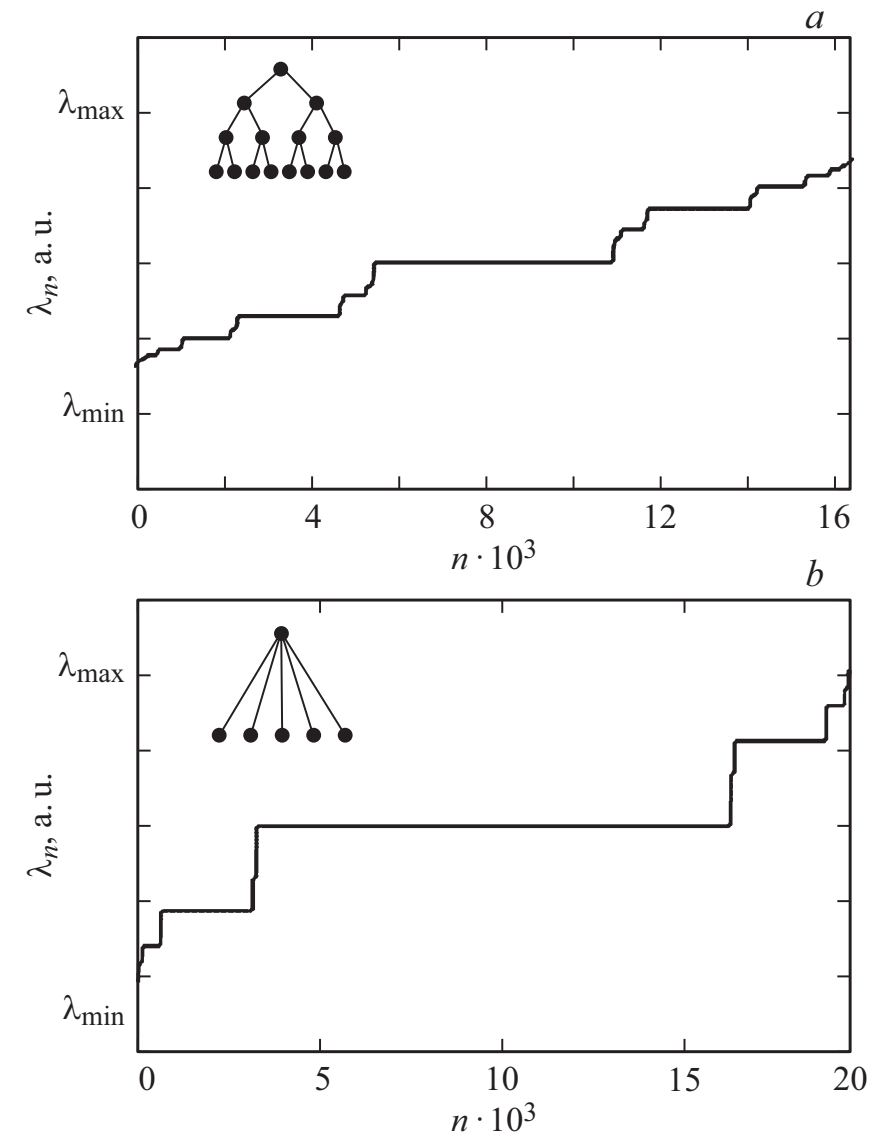

Рис. 2. Спектры нормальных мод $\lambda_{n}$ иерархических деревьев с числом ребер, равным двум $(a)$ и пяти $(b)$. 

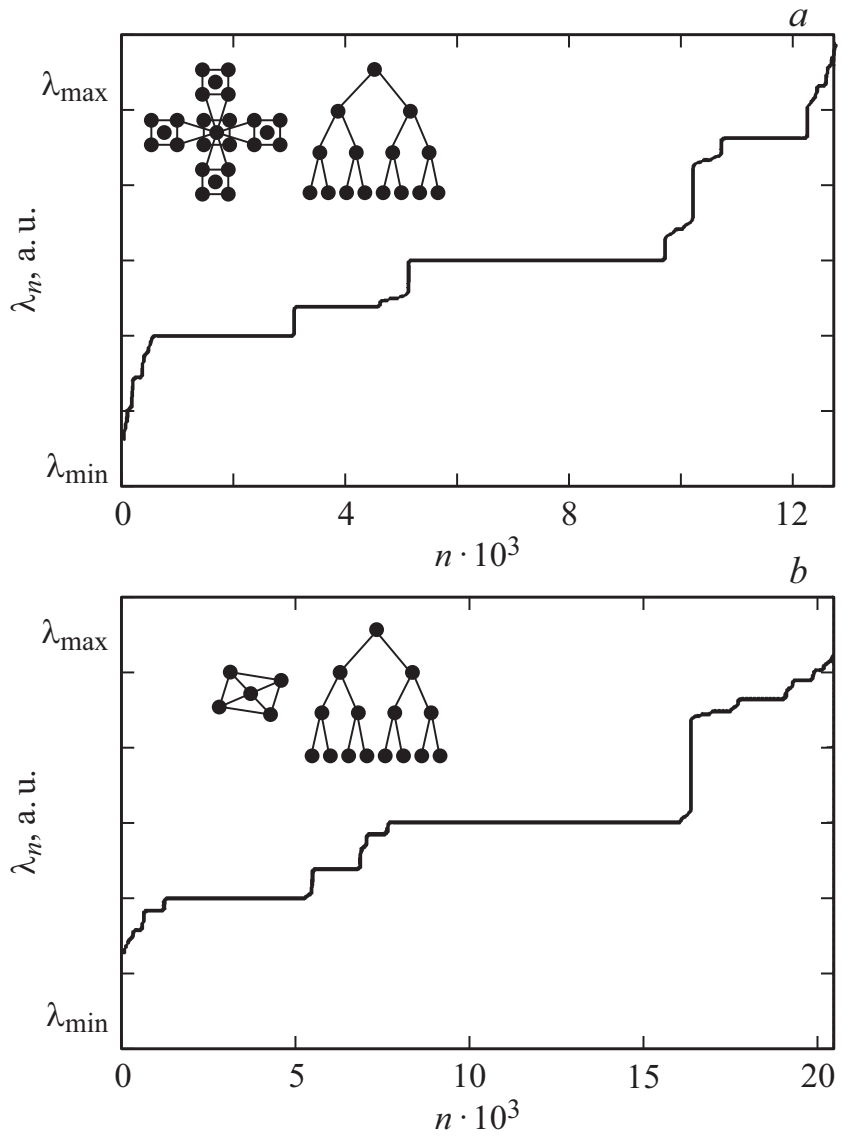

Рис. 3. Спектр нормальных мод $\lambda_{n}$ иерархического дерева с двумя ветвями, в узлах которого расположены разные метаструктуры: иерархический ансамбль $(a)$ и ансамбль вида „конверт“ $(b)$.

Многие сложные сети имеют частично иерархическую структуру, фрактальную в пределе, и ее простейшим типом является дерево. На рис. 2 представлены спектры нормальных мод $\lambda_{n}$ иерархических деревьев с двумя $\left(N=16 \cdot 10^{3}\right)$ и пятью $\left(N=20 \cdot 10^{3}\right)$ ребрами в случаях, когда в узлах расположены отдельные осцилляторы. При этом сила связей между всеми элементами дерева предполагалась одинаковой. Видно, что спектр дерева с двумя ребрами имеет форму фрактала типа „чертова лестница“" [7]. При увеличении числа ребер до пяти спектр „расползается“, сохраняя самоподобие. При этом наблюдается широкая область вырожденных мод на центральной частоте $\left(\lambda_{\max }-\lambda_{\min }\right) / 2$. Для сетей с другим количеством ребер фрактальный вид спектра сохраняется, однако количество вырожденных мод (горизонтальных „полок“ в спектре) будет разным. Для задач синхронизации сети с широкой областью вырожденных мод могут быть предпочтительнее сетей с „разреженным“ спектром.

Рассмотрим влияние метаструктур на характер спектров нормальных мод. На рис. 3 приведены спектры древовидных сетей с двумя ребрами с изображением метаструктур в узлах сети. Как видно, фрактальный характер спектров в этом случае частично сохраняется.
Однако влияние метаструктур сильно отражается на виде спектров. В частности, для неиерархической метаструктуры (рис. $3, b)$ наблюдается широкая область „запрещенных“" мод, т. е. частот, которые в системе не могут возбудиться. При значительном усилении связей между ребрами дерева спектр сложной сети приближается к спектру дерева, а в аналогичном случае усиления связей между осцилляторами метаструктур спектр сложной сети принимает форму спектра метаструктуры. Отсюда следует, что путем изменения формы и силы связи метаструктуры можно синтезировать необходимый вид спектра нормальных мод. Задача синтеза топологии сети по заданной структуре спектра нормальных мод (закон дисперсии для непрерывных систем) является предметом отдельного исследования и здесь не рассматривается.

Таким образом, в работе исследовано семейство нормальных мод иерархически организованных ансамблей идентичных взаимосвязанных осцилляторов с различной топологией организации и формами входящих метаструктур. Показано, что древовидные ансамбли обладают фрактальным спектром нормальных мод типа „чертова лестница“. Наличие иерархии в организации ансамбля ведет к вырожденности спектра нормальных мод, тогда как при рандомизации вырожденность в спектре пропадает. В дальнейшем планируется исследовать различные динамические процессы в таких сетях: синхронизацию, многомодовую генерацию, образование химерных состояний (см. подробнее [11-13]). Предлагаемый анализ может использоваться для создания фрактальных наноразмерных атомных сетей [14], магнитных наночастиц [15] и осцилляторов [16], а также устройств, основанных на новых физических принципах $[17,18]$.

\section{Финансирование работы}

Работа выполнена при финансовой поддержке базовой части государственного задания РФ № 8.8109.2017/БЧ.

\section{Конфликт интересов}

Авторы заявляют, что у них нет конфликта интересов.

\section{Список литературы}

[1] Arenas A., Diaz-Guilera A., Kurths J., Moreno Y., Zhou C. // Phys. Rep. 2008. V. 469. N 3. P. 93-153.

[2] Dörfler F., Bullo F. // Automatica. 2014. V. 50. N 6. P. 1539 1564.

[3] Endo T., Mori S. // IEEE Trans. Circuits Syst. 1976. V. 23. N 2. P. $100-113$.

[4] Endo T., Mori S. // IEEE Trans. Circuits Syst. 1976. V. 23. N 9. P. $517-530$.

[5] Endo T., Mori S. // IEEE Trans. Circuits Syst. 1978. V. 25. N 1. P. 7-18.

[6] Linkens D.A. // IEE Proc. A. 1983. V. 130. N 2. P. 69-87.

[7] Nakayama T., Yakubo T., Orbach R. // Rev. Mod. Phys. 1994. V. 66. N 2. P. 381-443. 
[8] Rammal R., Toulouse G., Virasoro M. // Rev. Mod. Phys. 1986. V. 58. N 3. P. 765-788.

[9] Аветисов В.А., Бикулов А.Х., Васильев О.А., Нечаев С.К., Чертович А.В. // ЖЭТФ. 2009. Т. 136. В. 3. С. 566-588.

[10] Козырев С.В. // ТМФ. 2015. Т. 185. № 2. С. 346-360.

[11] Крюков А.К., Осипов Г.В. // Изв. вузов. Прикладная нелинейная динамика. 2013. Т. 21. № 2. С. 188-200.

[12] Ахманов С.А., Воронщов М.A. // Нелинейные волны: динамика и эволюция: Сб. статей. М.: Наука, 1989. С. 228237.

[13] Алешин К.Н., Матросов В.В., Шалфеев В.Д. // Изв. вузов. Радиофизика. 2016. Т. 59. № 1. С. 55-66.

[14] Kempkes S.N., Slot M.R., Freeney S.E., Zevenhuizen S.J.M., Vanmaekelbergh D., Swart I., Morais Smith C. // Nature Phys. 2018. V. 15. N 2. P. 127-131.

DOI: $10.1038 / \mathrm{s} 41567-018-0328-0$

[15] Osokin S.A., Safin A.R., Barabanenkov Y.N., Nikitov S.A. // J. Magn. Magn. Mater. 2018. V. 465. P. 519-523.

[16] Safin A.R., Udalov N.N., Kapranov M.V. // Eur. Phys. J. Appl. Phys. 2014. V. 67. N 2. P. 20601.

[17] Карпасюк В.К., Булатов М.Ф. // ФТТ. 1999. Т. 41. В. 11. C. 2016-2019.

[18] Karpasyuk V.K., Bulatov M.F. // IEEE Trans. Magn. 1994. V. 30. N 6. P. $4344-4346$. 